\title{
PCR mediated recombination impacts the analysis of hepatitis B Virus covalently closed circular DNA
}

\author{
Rodolphe Suspène, Valérie Thiers, Jean-Pierre Vartanian* and Simon Wain-Hobson ${ }^{*}$
}

\begin{abstract}
Background: The replication of HBV involves the production of covalently closed circular DNA (cccDNA) from the HBV genome through the repair of virion relaxed circular DNA (rcDNA) in the virion. As cccDNA is the transcription template for HBV genomes, it needs to be eliminated from hepatocytes if the eradication of chronic HBV infection is to be achieved. PCR quantitation of cccDNA copy number is the technique of choice for evaluating the efficiency of treatment regimens. The PCR target commonly used to identify cccDNA spans the gapped region of rcDNA and is considered to accurately distinguish between cccDNA and rcDNA. There is however, a potentially confounding issue in that PCR can generate larger targets from collections of small DNA fragments, a phenomenon known as PCR recombination.

Results: The impact of PCR recombination towards the amplification of this cccDNA specific target was explored by mixing three marked, yet overlapping HBV DNA fragments. Thirteen of sixteen possible recombinants were identified by sequencing with frequencies ranging from 0.6 to $23 \%$. To confirm this finding in vivo, HBV positive sera were treated with DNase I and submitted to quantitative real-time PCR. Under these conditions, it was possible to amplify the cccDNA specific segment without difficulty. As the virion contains uniquely rcDNA, amplification of the cccDNA target resulted from PCR recombination.
\end{abstract}

Conclusions: PCR quantitation of cccDNA may be more difficult than hitherto thought. Current detection protocols need to be investigated so as to help in the management of chronic HBV infection.

Keywords: HBV, ccCDNA, rCDNA, PCR recombination

\section{Findings}

Incoming partly double stranded hepatitis B virus (HBV) DNA genomes are completed by the viral polymerase generating relaxed circular DNA (rcDNA). Following translocation to the nucleus, the gaps are repaired by the host repair system to generate covalently closed circular DNA (cccDNA), the template for the HBV pregenome and viral mRNAs. Consequently, catabolism of cccDNA is necessary for the eradication of chronic HBV infection. cccDNA levels are a useful biomarker of HBV replication although its clinical use is restricted as it requires

\footnotetext{
${ }^{*}$ Correspondence: jean-pierre.vartanian@pasteur.fr; simon.wain-hobson@pasteur.fr

Molecular Retrovirology Unit, Institut Pasteur, 28 rue du Dr. Roux, 75724 Paris Cedex 15, France
}

invasive sampling. PCR quantitation of cccDNA is widely used to assess the effectiveness of antiviral therapy or in the analysis of anti-HBV restriction factors [1-4].

As the amount of HBV rcDNA in the cell is much higher than cccDNA, distinguishing the latter is a challenge. The signal differences between the two forms are the two gaps in rcDNA. PCR across this region has been considered to selectively amplify cccDNA. In the later cycles of a PCR reaction, where $[\mathrm{DNA}]>[\mathrm{Taq}]$, not all strands are completed. This is highlighted by addition of a 10 min step at the end of the reaction. During these later cycles, incomplete DNA strands can switch templates resulting in the formation of recombinant DNA strands, a phenomenon referred to as PCR mediated recombination [5]. This is not detectable when the 
target is genetically homogenous but is readily seen for HIV where there is considerable intra-sample genetic heterogeneity [6]. Similarly "DNA shuffling" generates large recombinant DNA molecules from smaller DNA fragments through template switching [7]. Here strand switching occurs by the second round of PCR and larger single stranded DNA molecules are built up extending from the outer primers until they overlap. From that point on classical PCR takes over. The amplification HBV cccDNA is akin to DNA shuffling.

We wondered whether PCR mediated recombination during amplification of the double gapped region of HBV rcDNA could generate the target sequence considered to be specific for cccDNA.

To test this, three different overlapping HBV fragments simulating the double gapped structure of rCDNA were PCR amplified (PCR1 positions 1545-1818; PCR2 positions 1765-1887; PCR3 position 1819-1954; accession number NC_003977.2; Fig. 1a). Primers incorporated mutations (underlined) to distinguish PCR fragments from input HBVayw DNA. Primers were A1sens, 5'GACAGCCC GTCTGTGCCTTCTCATGACCCGGA and A2rev, 5'CATGCAGCTGGTGCGCAGACCAA for PCR1, B1sens 5'GGTCTAAGTACTAGGAGGCTGTA and B2rev 5'AAGGCTGAGCTTGGAGGCTTGAA for PCR2 and C1sens 5'CAACAATTTCACCTCTGCC TAAT and C2rev 5'ACGTCAGTAACTCCACAGT AGCAGGAAATTCT for PCR3. Amplification conditions were identical for the three fragments and corresponded to $5 \mathrm{~min}$ at $95^{\circ} \mathrm{C}$ then $30 \mathrm{~s}$ at $95^{\circ} \mathrm{C}, 30 \mathrm{~s}$ at $58^{\circ} \mathrm{C}$ and $2 \mathrm{~min}$ at $72{ }^{\circ} \mathrm{C}$ for 35 cycles, being completed by a final incubation at $72{ }^{\circ} \mathrm{C}$ for $10 \mathrm{~min}$. Reactions were performed in a final volume of $100 \mu \mathrm{l}$.

The three PCR fragments were purified from agarose gels (Qiaex II kit, Qiagen, France), quantified by nanodrop spectrophotometer, mixed on an equimolar basis (100 ng final) and subjected to 35 cycles of amplification with the outer primers A1tsens, 5'GACAG CCCGTCTGTGCCTTCTCATGAC and C2trev,

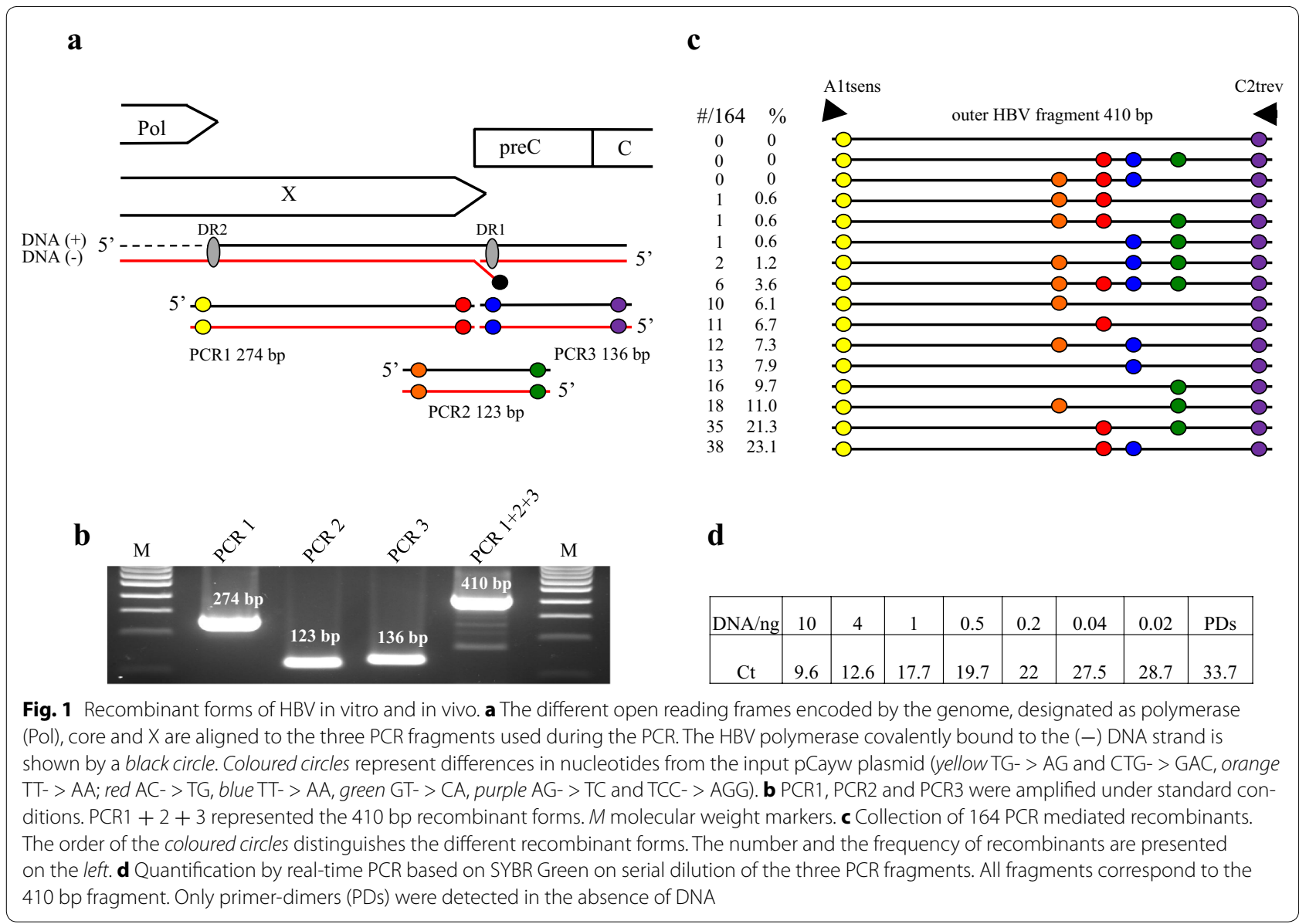


5'ACGTCAGTAACTCCACAGTAGCAGG using the above PCR conditions. Primers A1t and C2t corresponded to primers $\mathrm{A} 1$ and $\mathrm{C} 2$ and were shortened respectively by 5 and 7 nucleotides $3^{\prime}$ of the primer to avoid amplification of HBVayw plasmid. As can be seen from Fig. 1b, a 410 bp fragment was readily obtained from the mixture of the 123, 136 and $274 \mathrm{bp}$ fragments. The $410 \mathrm{bp}$ outer PCR products were cloned using the TOPO TA cloning kit (Invitrogen) and individual clones sequenced.

As can be seen in Fig. 1c, all of the 164 sequences were recombinants where coloured circles represent the mutations introduced into the primers. Thirteen of the sixteen possible recombinants were found with frequencies ranging from 0.6 to $23 \%$. The marker mutations, identified by colour in Fig. 1a, were present in roughly the same proportions across the 164 sequences consistent with the fact that equimolar proportions of each DNA fragment were used (orange $\sim 30 \%$, red $\sim 56 \%$, blue $\sim 44 \%$ and green $\sim 48 \%)$. Some PCR mediated recombinants required just two template switches (one circle) while others required six (4 circles, Fig. 1c). The most complex recombinantfour circles-represented $3.6 \%$ of the total. Interestingly, no clones with the HBVayw reference sequence were found (no circles, Fig. 1a) meaning that there was no carry over of input HBV DNA during purification of the amplicons.

To determine the sensitivity of the PCR, we performed real-time PCR based on SYBR Green with serial dilutions of an equimolar mix of the three PCR fragments. Amplification conditions corresponded to $10 \mathrm{~min}$ at $95{ }^{\circ} \mathrm{C}$ then $20 \mathrm{~s}$ at $95{ }^{\circ} \mathrm{C}, 20 \mathrm{~s}$ at $55^{\circ} \mathrm{C}$ and 2 min at $68{ }^{\circ} \mathrm{C}$ for 40 cycles with primers A1tsens and C2trev. Amplification was completed by a melting curve step and all products were analysed by gel electrophoresis. As can be seen from Fig. 1d, 410 bp PCR recombinants could be detected down to $20 \mathrm{pg}$ input DNA. While this is a rather high threshold, PCR recombination involves the diffusion of large molecules many times larger than typical amplification primers. In addition, a minimum of three cycles are necessary to assemble the $410 \mathrm{bp}$ fragment. Hence, a rather elevated detection threshold is to be expected.

In order to demonstrate PCR mediated recombination of bona fide HBV rcDNA, sera from $4 \mathrm{HBV}$ infected patients (S1-S4) with high HBV copy numbers (S1 8.42; S2 7.28; S3 8.72, S4 $7.53 \log$ UI previously described [8]) were analysed. Serum can harbour circulating genomic DNA and so may contain a little cccDNA from necrotized hepatocytes. To investigate this, sera were split and part was treated with DNase I (5 units, $2 \mathrm{~h}$, $37{ }^{\circ} \mathrm{C}$, heat-inactivated at $95{ }^{\circ} \mathrm{C}, 10 \mathrm{~min}$, Fig. 2a). TP53 DNA was targeted using primers P53sens $5^{\prime}$ GAGC TGGACCTTAGGCTCCAGAAAGGACA and P53rev 5'GCTGGTGTTGTTGGGCAGTGCTAGGAA, PCR conditions were described [9] while HBV DNA was amplified across the double gap structure using primers HBVsens 5'GACTCCCCGTCTGTGCCTTCTCA and HBVrev 5'ACGAGAGTAACTCCACAGTAGCT. TP53 DNA could be recovered from untreated, but not DNase I treated sera proving the presence of circulating chromosomal DNA in all four samples. By contrast HBV DNA could be found in both DNase I treated and untreated sera (Fig. 2a). Presumably the HBV signal from the treated sample comes from rcDNA protected by the virion. DNase I treated HBV PCR products were cloned and 15-20 clones sequenced from each sample (Fig. 2b). Sequences were identical within a serum sample but all different between samples proving no cross contamination between samples. As the virion uniquely contains rcDNA, amplification of a $410 \mathrm{bp}$ fragment inevitably required PCR mediated recombination.

To investigate sensitivity, we performed real-time SYBR Green PCR. Serum S1 (viral load of $8.42 \mathrm{log}$ IU/ $\mathrm{ml}$ ) was digested with DNase I, serially diluted and the $\mathrm{Ct}$ determined. There was a good linear correlation between $\log [\mathrm{DNA}]$ and Ct (Fig. 2c) indicating that PCR recombinants could be obtained down to the limits of detection, which is a little lower than 3600 copies. Protocols for quantifying cccDNA usually include a treatment by a Plasmid-Safe DNase. Accordingly, DNase I treated sera S1-S3 were heat inactivated and treated with PlasmidSafe DNase (3 units, Illumina) or with T4 Exonuclease (3 units, New England BioLabs) for $30 \mathrm{~min}$ at $37^{\circ} \mathrm{C}$ which is typical of such protocols [10]. Target cccDNA was quantitated by SYBR Green PCR. As can be observed in Fig. 2d, treatment with Plasmid-Safe DNase resulted in a higher $\mathrm{Ct}$ value indicating that treatment had eliminated input DNA. T4 exonuclease treatment eliminated even more DNA (Fig. 2d). Despite these treatments, $410 \mathrm{bp}$ target cccDNA could be recovered by PCR recombination, for the input was uniquely rcDNA.

As HBV cccDNA is synonymous with persistent infection, it is both a therapeutic target and an attractive biomarker to follow, while PCR is the obvious technology to employ. Distinguishing rcDNA and cccDNA in the nucleus is extremely difficult. That the former is found in the nucleus is indicated by integrated HBV sequences with integration sites mapping close to the $5^{\prime}$ ends of 
a

DNase I M $\frac{\mathrm{S} 1}{+-} \frac{\mathrm{S} 2}{+-} \frac{\mathrm{S} 3}{+-} \frac{\mathrm{S} 4}{+-\mathrm{M}}$

TP53

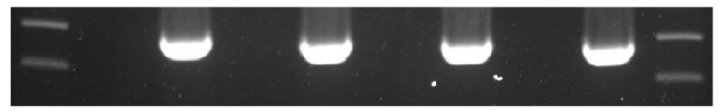

DNase I $\quad+\quad-\quad+\quad-\quad+\quad-\quad+\quad-$

HBV

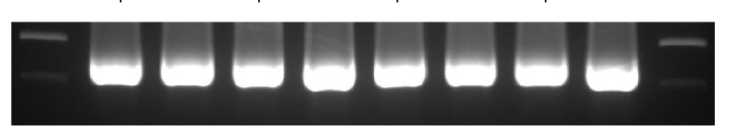

b

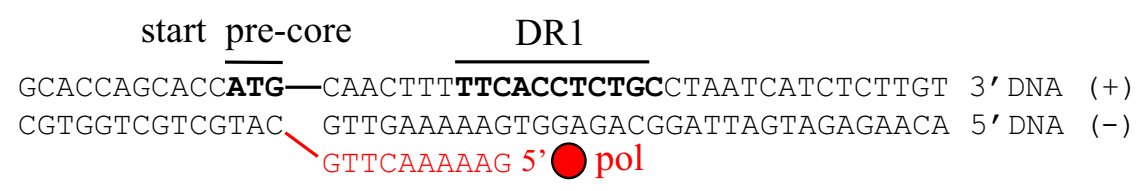

TCTGCGCACCAGCACCATG CAACTTTTTCACCTCTGCCTAATCATCTCTTGTTCATGTCCTACTGTTCAAGCCTCCA S1 x15

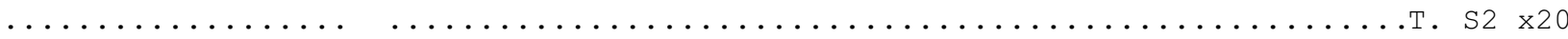

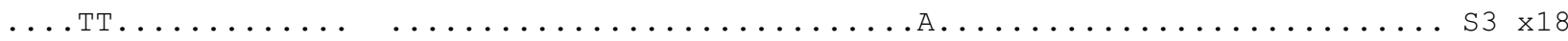

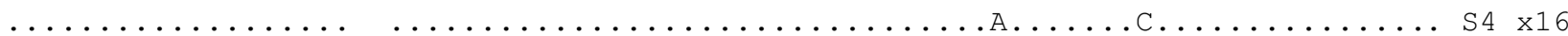

\section{c}

d

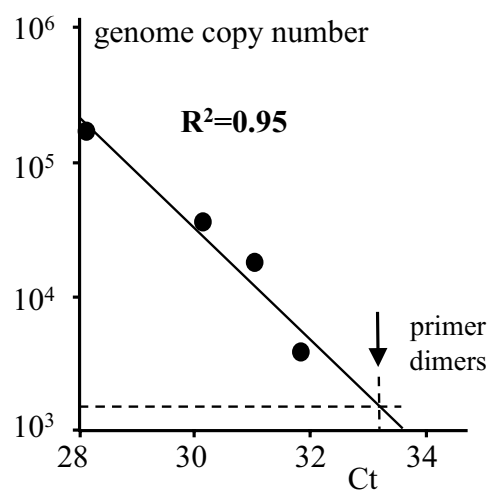

\begin{tabular}{|l|c|c|c|}
\cline { 2 - 4 } \multicolumn{1}{c|}{} & \multicolumn{3}{c|}{ Ct } \\
\cline { 2 - 4 } \multicolumn{1}{c|}{} & $\mathrm{S} 1$ & $\mathrm{~S} 2$ & $\mathrm{~S} 3$ \\
\hline DNase & 27.5 & 29.0 & 29.2 \\
\hline DNase + PLMD DNase & 30.4 & 30.7 & 30.9 \\
\hline DNase + T4 exonuclease & 31.1 & 32.3 & 32.1 \\
\hline Primer-dimers & \multicolumn{3}{|c|}{33.2} \\
\hline
\end{tabular}

Fig. 2 Target ccCDNA generated from virion associated rCDNA. a PCR analysis of the serum of 4 HBV infected patients (S1-S4) in presence or absence of DNase I treatment. TP53 amplification (top) and HBV (bottom). b Sequences of HBV from the serum of the 4 infected patients. The sequence from serum 1 was used as reference, the number of sequences analysed is given on the right. Above is a representation of rcDNA for this region. c Quantification by real-time PCR based on SYBR Green on serum S1. d SYBR Green PCR quantification after DNase treatment followed by Plasmid-safe DNase and T4 exonuclease on serum S1, S2 and S3. All amplifications were controlled by gel electrophoresis. Above a Ct of 32.3 only primer-dimers were recovered

rcDNA strands [11]. From this point on, PCR mediated recombination becomes an issue for the identification of cccDNA. The use of longer elongation times reduces but does not eliminate PCR recombination [5]. Unambiguous quantitation of cccDNA by PCR may be more difficult than hitherto thought.

\section{Authors' contributions}

RS, VT, JPV and SWH analysed the data and wrote the manuscript. All authors read and approved the manuscript.

\section{Acknowledgements}

We thank Stanislas Pol for providing the sera.

\section{Competing interests}

The authors declare that they have no competing interests.

\section{Funding}

This work was funded by the Institut Pasteur and the Centre National de Recherche Scientifique (CNRS).

Received: 5 October 2016 Accepted: 24 November 2016 Published online: 20 December 2016 


\section{References}

1. Margeridon S, Carrouee-Durantel S, Chemin I, Barraud L, Zoulim F, Trepo C, Kay A. Rolling circle amplification, a powerful tool for genetic and functional studies of complete hepatitis B virus genomes from low-level infections and for directly probing covalently closed circular DNA. Antimicrob Agents Chemother. 2008;52:3068-73.

2. Mu D, Yan L, Tang H, Liao Y. A sensitive and accurate quantification method for the detection of hepatitis B virus covalently closed circular DNA by the application of a droplet digital polymerase chain reaction amplification system. Biotechnol Lett. 2015;37:2063-73.

3. Werle-Lapostolle B, Bowden S, Locarnini S, Wursthorn K, Petersen J, Lau G, Trepo C, Marcellin P, Goodman Z, Delaney WE, et al. Persistence of cccDNA during the natural history of chronic hepatitis $B$ and decline during adefovir dipivoxil therapy. Gastroenterology. 2004;126:1750-8.

4. Lucifora J, Xia Y, Reisinger F, Zhang K, Stadler D, Cheng X, Sprinzl MF, Koppensteiner H, Makowska Z, Volz T, et al. Specific and nonhepatotoxic degradation of nuclear hepatitis B virus cccDNA. Science. 2014;343:1221-8.

5. Meyerhans A, Vartanian JP, Wain-Hobson S. DNA recombination during PCR. Nucleic Acids Res. 1990;18:1687-91.

6. Yang YL, Wang G, Dorman K, Kaplan AH. Long polymerase chain reaction amplification of heterogeneous HIV type 1 templates produces recombination at a relatively high frequency. AIDS Res Hum Retroviruses. 1996;12:303-6.
7. Stemmer WP. Rapid evolution of a protein in vitro by DNA shuffling Nature. 1994;370:389-91.

8. Miailhes P, Maynard-Muet M, Lebosse F, Carrat F, Bouix C, Lascoux-Combe C, Sogni P, Rey D, Barthe Y, Pol S, et al. Role of a 48-week pegylated interferon therapy in hepatitis B e antigen positive HIV-co-infected patients on CART including tenofovir: EMVIPEG study. J Hepatol. 2014;61:761-9.

9. Suspène R, Aynaud M, Guétard D, Henry M, Eckhoff G, Marchio A, Pineau P, Dejean A, Vartanian JP, Wain-Hobson S. Somatic hypermutation of human mitochondrial and nuclear DNA by APOBEC3 cytidine deaminases, a pathway for DNA catabolism. Proc Natl Acad Sci USA. 2011;108:4858-63.

10. Gao YT, Han T, Li Y, Yang B, Wang YJ, Wang FM, Jing X, Du Z. Enhanced specificity of real-time PCR for measurement of hepatitis $B$ virus cccDNA using restriction endonuclease and plasmid-safe ATP-dependent DNase and selective primers. J Virol Methods. 2010;169:181-7.

11. Dejean A, Brechot C, Tiollais P, Wain-Hobson S. Characterization of integrated hepatitis B viral DNA cloned from a human hepatoma and the hepatoma-derived cell line PLC/PRF/5. Proc Natl Acad Sci USA. 1983;80:2505-9.

\section{Submit your next manuscript to BioMed Central and we will help you at every step:}

- We accept pre-submission inquiries

- Our selector tool helps you to find the most relevant journal

- We provide round the clock customer support

- Convenient online submission

- Thorough peer review

- Inclusion in PubMed and all major indexing services

- Maximum visibility for your research

Submit your manuscript at www.biomedcentral.com/submit 